\title{
Long non-coding RNA homeobox (HOX) A11-AS promotes malignant progression of glioma by targeting miR-124-3p
}

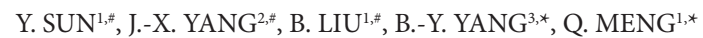 \\ ${ }^{1}$ Department of Neurosurgery, Tangdu Hospital, Fourth Military Medical University, Xian 710038, China; ${ }^{2}$ Department of Neurosurgery, The \\ Second People Hospital of Xin Xiang, Xin Xiang 453003, China; ${ }^{3}$ Department of Neurosurgery, 999 Brain Hospital of Guang Dong, Guang Zhou \\ 510510, China
}

*Correspondence: mengqiang_mq@aliyun.com,baoying2012_yang@21cn.com

${ }^{*}$ Contributed equally to this work.

Received July 5, 2017 / Accepted September 27, 2017

\begin{abstract}
Glioma is the most common and serious form of primary tumor in adult central nervous system. HOXA11-AS is a LncRNA located in the HOXA gene cluster. In the present study, we investigated the expression and function of LncRNA HOXA11-AS in glioma tissues and cells. We found that LncRNA HOXA11-AS expression was markedly elevated in glioma tissues compared to normal brain tissues. The LncRNA HOXA11-AS expression in cases of high-grade glioma was significantly higher than that in cases of low-grade. Patients with high LncRNA HOXA11-AS expression had shorter OS time than those with low LncRNA HOXA11-AS expression. Moreover, silencing LncRNA HOXA11-AS inhibited cell proliferation, increased apoptosis, and inhibited invasion and migration of glioma cells. Overexpression of LncRNA HOXA11AS increased cell proliferation, decreased apoptosis, and increased invasion and migration of glioma cells. miR-124-3p has relevant binding sites in HOXA11-AS. Silencing HOXA11-AS significantly increased miR-124-3p expression. The miR-124-3p overexpression decreased the luciferase activity of the pMIR luciferase reporter containing HOXA11-AS-WT but not HOXA11-AS-MUT. Moreover, miR-124-3p was pulled down by HOXA11-AS probe. miR-124-3p mimics inhibited cell proliferation, increased apoptosis, and inhibited invasion and migration of glioma cells. miR-124-3p mimics significantly suppressed overexpression of HOXA11-AS-induced increase of proliferation, decrease of apoptosis and increase of invasion and migration. miR-124-3p inhibitors suppressed the effect of siHOXA11-AS on proliferation, apoptosis, invasion and migration. In summary, the findings highlight the importance of LncRNA HOXA11-AS/miR-124-3p axis in the regulation of glioma progression. LncRNA HOXA11-AS/miR-124-3p might serve as a potential therapeutic target in glioma treatment in the future.
\end{abstract}

Key words: glioma, LncRNA HOXA11-AS, miR-124-3p, proliferation, invasion, migration

Glioma is the most common and serious form of primary tumor in adult central nervous system (CNS). Despite advances in combination treatments, including surgical resection followed by radiation therapy and chemotherapy, the average survival of malignant glioma patients has been only slightly improved in the past decades with the median survival of about 1 year [1-4]. Therefore, identifying new molecular biomarkers and finding novel therapeutic strategies are of great importance for effective glioma treatment.

The long non-coding RNAs (LncRNAs) were once considered to be a transcriptional noise with no function [5]. However, in recent years, it has been identified that the vast majority (98\%) of the human genome can be transcribed into non-coding RNAs [6]. Accumulating evidence has demonstrated that LncRNAs play critical roles in various biological processes, including transcriptional regulation [7], proliferation [8], differentiation [9], immune response [10], metabolism [11]. In recent years, the roles of LncRNAs in the regulation of tumorigenesis have acquired extensive attention $[12,13]$. LncRNAs have been believed to function as key molecules in cancer development and progression $[14,15]$. Many LncRNAs have been reported to serve as oncogenes or tumor suppressors $[16,17]$.

Homeobox (HOX) are a family of genes that act as important transcriptional factors during embryogenesis and carcinogenesis [18]. HOX proteins possess highly conserved homeodomains that enable them to bind to specific DNA regions and activate or repress transcription of target genes 
[19]. In human, HOX genes are classified into four clusters, including $\mathrm{A}, \mathrm{B}, \mathrm{C}$, and $\mathrm{D}$, which are located on four different chromosomes [20]. HOXA11-AS is a LncRNA located in the HOXA gene cluster. Aberrant expression of HOXA11-AS has been reported to be involved in cancer progression [21-23]. However, the functional role of HOXA11-AS in the glioma is still not well clarified and needs to be further elucidated.

In the present study, we investigated the expression and function of LncRNA HOXA11-AS in glioma tissues and cells. We found that LncRNA HOXA11-AS was upregulated in glioma tissues and cells and was associated with malignancy and poor prognosis of glioma patients. There was a specific binding site between LncRNA HOXA11-AS and miR-124-3p. LncRNA HOXA11-AS and miR-124-3p negatively regulated their respective expression. Silencing LncRNA HOXA11-AS inhibited proliferation, increased apoptosis, decreased invasion and migration ability of glioma cells. Overexpression of miR-124-3p inhibited the promoting effect of LncRNA HOXA11-AS on malignancy in glioma cells. These findings highlight the importance of LncRNA HOXA11-AS/miR-124-3p in the regulation of glioma progression and suggest that LncRNA HOXA11-AS/ miR-124-3p might be a novel therapeutic target.

\section{Patients and methods}

Patients and tissue specimens. A total of 71 glioma tissues (38 astrocytomas, 12 oligodendrogliomas, 6 ependymomas, 4 choroid plexus tumors, and 11 others) were obtained from patients who had undergone surgical treatment at the Tangdu Hospital, Fourth Military Medical University between 2015 and 2016. The patients did not receive any radiotherapy or chemotherapy before surgery. A total of 15 normal brain tissues from patients with cerebral trauma or cerebral hemorrhage were cut and used as control. All patients had experienced a follow-up period lasting 48 months from the date of surgical resection. Overall survival (OS) was defined as the time from the date of initial surgical operation to the date of death or the date of the last follow-up if death did not occur. Informed consent was obtained from all participating patients. All procedures were carried out in accordance with the Internal Review and the Ethics Board of Tangdu Hospital, Fourth Military Medical University. The Ethics Committee of Tangdu Hospital, Fourth Military Medical University approved the study.

Cell culture and transfection. U87, U251, and T98G human glioma cell lines were purchased from the Cell Bank of the Shanghai Branch of Chinese Academy of Sciences. Cells were cultured in Dulbecco's Modified Eagle's Medium (DMEM; Gibco, USA) supplemented with $10 \%$ fetal bovine serum (FBS; Gibco, USA). The cells were maintained in a humidified incubator at $37^{\circ} \mathrm{C}$ with $5 \% \mathrm{CO}_{2}$. HOXA11AS-specific small interfering RNA (named siHOXA11AS, sequence: CGAAAGCACGUAAUCGCCGGUGUAA) targeting HOXA11-AS and negative control (NC, a nonspe- cific scramble siRNA), and pcDNA HOXA11-AS and control vectors, miR-124-3p mimics and inhibitors were synthesized commercially (GenePharma, Shanghai, China). For the transfection, the cells were $60 \%$ confluent and then transfected with $100 \mathrm{nM}$ siRNA or $100 \mathrm{nM}$ miRNA mimics and inhibitors using Turbofect reagent following the manufacturer's instructions (Thermo Fisher Scientific, Rockford, IL, USA). Cells were collected $48 \mathrm{~h}$ after transfection. Then, the transfection efficacy was examined by quantitative real-time polymerase chain reaction (qRT-PCR) analysis.

Cell proliferation. After the transfection, cell proliferation was evaluated by CCK8 assay kit. Briefly, the cell culture medium was removed after the treatment and fresh FBS-free medium was added and then CCK8 assay solution was added. After incubation for $2 \mathrm{~h}$ at $37^{\circ} \mathrm{C}$, absorbance at 450 $\mathrm{nm}$ was determined.

Apoptosis. Cell apoptosis was detected by an In situ Apoptosis Detection Assay kit (Roche, Switzerland). After the treatment, the cells were trypsinized and then suspended in reaction solution and stained with TUNEL. After washing with PBS, cells were analyzed using a FACScan flow cytometer (BD Biosciences, USA).

Invasion assays. Cell invasion assay was carried out using the 24-well transwell chamber (Corning, USA) coated with Matrigel (BD Biosciences, USA). Transfected cells were washed, trypsinized, and seeded into the upper chamber in $100 \mu \mathrm{L}$ serum-free DMEM. The bottom chamber was filled with DMEM containing $10 \%$ FBS. After incubation at $37^{\circ} \mathrm{C}$ for $24 \mathrm{~h}$, the non-invasive cells on the top chambers were gently removed with cotton wool. Invasive cells on the bottom surface were fixed with $4 \%$ paraformaldehyde for $0.5 \mathrm{~h}$ and stained with $0.1 \%$ crystal violet containing $10 \%$ methanol for $2 \mathrm{~h}$. The number of invasive cells on the bottom surface was counted under a light microscope. Invasion was quantified by taking mean and standard deviations of 5 non-biased image fields. The experiments were performed in triplicates and results were averaged.

Wound healing assay. When the transfected cells reached $80-90 \%$ density, a wound was made using a $1 \mathrm{ml}$ plastic pipette tip before being washed twice with PBS. After that, cells were cultured in DMEM with 2\% FBS for $24 \mathrm{~h}$. The size of the wound was measured under a microscope at 0 and $24 \mathrm{~h}$ after wounding. Relative size of wound was calculated. The experiments were performed in triplicates and results were averaged.

Quantitative real-time PCR. Total RNA was extracted from brains using a RNA isolation kit and then reversely transcribed into cDNA using a miScript II RT Kit (Thermo Fisher Scientific Inc., Waltham, MA, USA), according to the manufacturer's instructions. Quantitative real-time polymerase chain reaction (PCR) was used to precisely quantify target genes by gene-specific primer pairs on a CFX96 Real-Time PCR Detection System (BioRad, CA, USA) using a miScript SYBR Green PCR Kit (Thermo Fisher Scientific Inc.). The reactions were as follows: an initial step 
at $94^{\circ} \mathrm{C}$ for $5 \mathrm{~min}$, followed by 40 cycles of denaturation at $94^{\circ} \mathrm{C}$ for $30 \mathrm{~s}$, annealing at $63^{\circ} \mathrm{C}$ for $30 \mathrm{~s}$, and then extension at $72^{\circ} \mathrm{C}$ for $10 \mathrm{~s}$. The $2^{-\Delta \Delta \mathrm{CT}}$ method was used to measure gene expression.

Dual-luciferase reporter assay. The luciferase assays were performed using the Dual-luciferase reporter assay system (Promega, USA). Briefly, cells were co-transfected with miR-124-3p mimics or miR-NC and pMIR-report luciferase vector containing 30-UTR of wild-type (WT) or mutant (MUT) HOXA11-AS fragment using Turbofect reagent following the manufacturer's instructions (Thermo Fisher Scientific, Rockford, IL, USA). 48 hafter transfection, cells were collected and lysed for luciferase detection. The relative luciferase activity was normalized to the Renilla luciferase activity.

Statistical analysis. The data were analyzed using SPSS17.0 statistical package (SPSS Inc., Chicago, IL, USA) and are presented as mean \pm standard deviation (SD) from at least three separate experiments. Differential expression of LncRNA HOXA11-AS between glioma and normal brain tissues was detected by independent two samples t test. OS was evaluated by the Kaplan-Meier analysis and log-rank test was used to compare the difference between groups. $\mathrm{P}$ values less than 0.05 were considered statistically significant.

\section{Results}

Overexpression of LncRNA HOXA11-AS correlates with histological malignancy of glioma and poor prognosis of glioma patients. To investigate the possible role of LncRNA HOXA11-AS in the glioma development and progression, we first evaluated its expression level in glioma tissues $(n=71)$ and normal brain tissues $(n=15)$ by qRT-PCR. We found that LncRNA HOXA11-AS expression was markedly elevated in glioma tissues compared to normal brain tissues (Figure 1A). Furthermore, we explored the relationship between LncRNA HOXA11-AS expression and histological malignancy in 38 low-grade (I-II) and 33 high-grade (III-IV) glioma tissues. In Figure 1B, we show that the LncRNA HOXA11-AS expression was significantly higher in high-grade than in low-grade gliomas. These results indicate that with the increase of
A

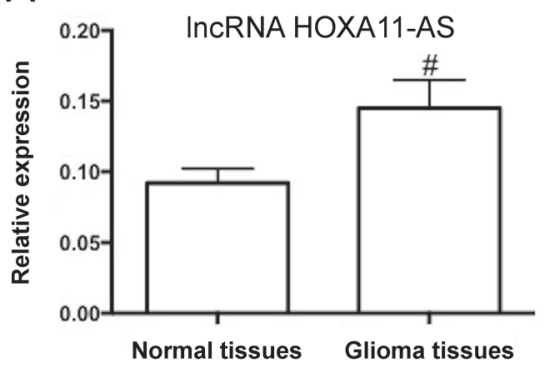

B

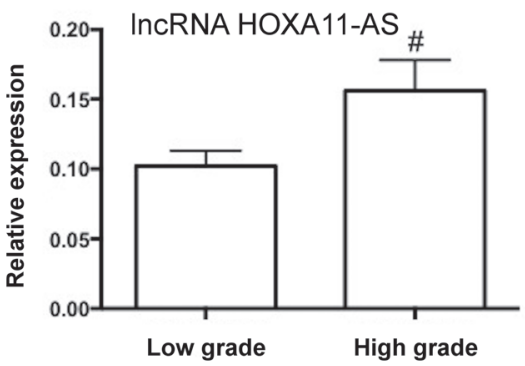

C

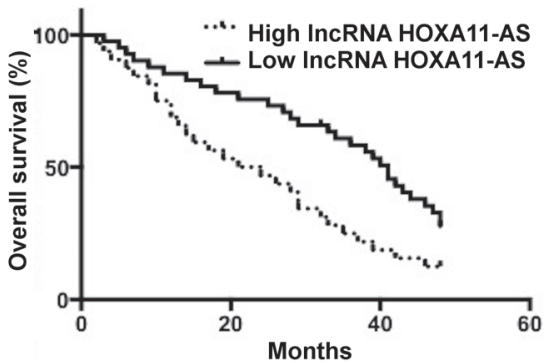

Figure 1. Upregulation of LncRNA HOXA11-AS was correlated with poor prognosis in glioma patients. (A) LncRNA HOXA11-AS expression in 71 human glioma tissues and 15 normal brain tissues was measured by qRT-PCR. (B) The difference in LncRNA HOXA11-AS expression in low-grade (I-II) (32) and high-grade (III-IV) (39) glioma tissues. (C) The patients with glioma were divided into LncRNA HOXA11-AS high-expression (33) and low-expression (38) group and Kaplan-Meier analysis was performed to evaluate the relationship between LncRNA HOXA11-AS expression level and prognosis of glioma patients $(\mathrm{p}=\mathbf{0 . 0 0 7 8 )}$. $\# \mathrm{p}<0.05$, compared with control.

A

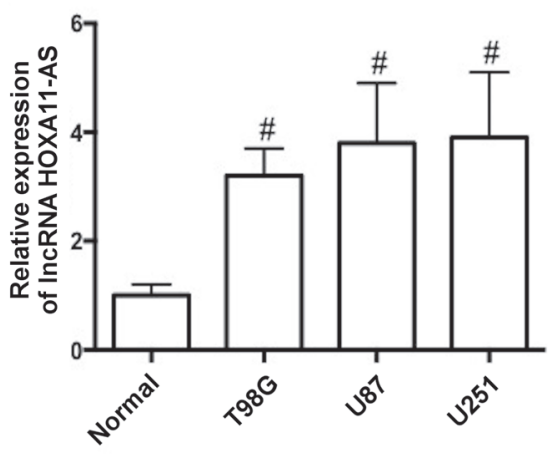

B

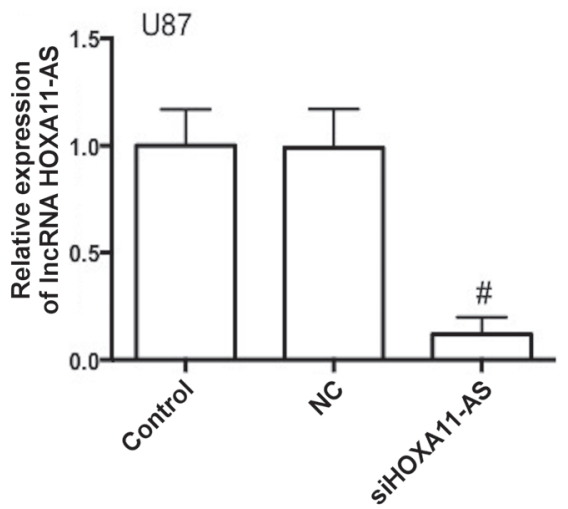

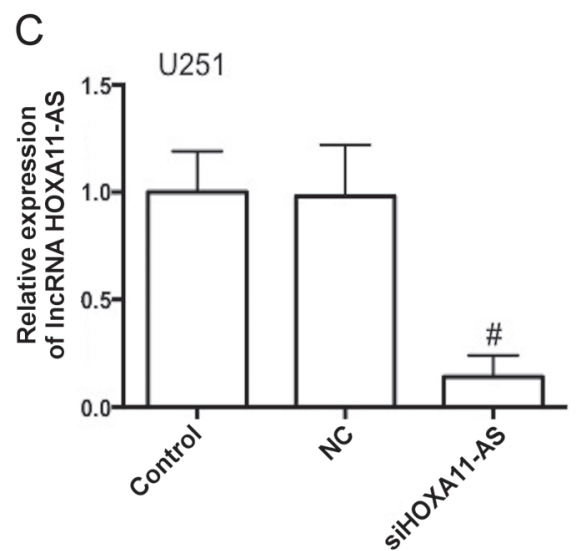

Figure 2. Silencing LncRNA HOXA11-AS expression in U87 and U251 cells by siHOXA11-AS. (A) LncRNA HOXA11-AS expression in normal brain and three glioma cell lines as determined by qRT-PCR. (B and C) Transfection of siHOXA11-AS reduced the HOXA11-AS expression in U87 and U251 cells. \# $\mathrm{p}<0.05$, compared with control. 

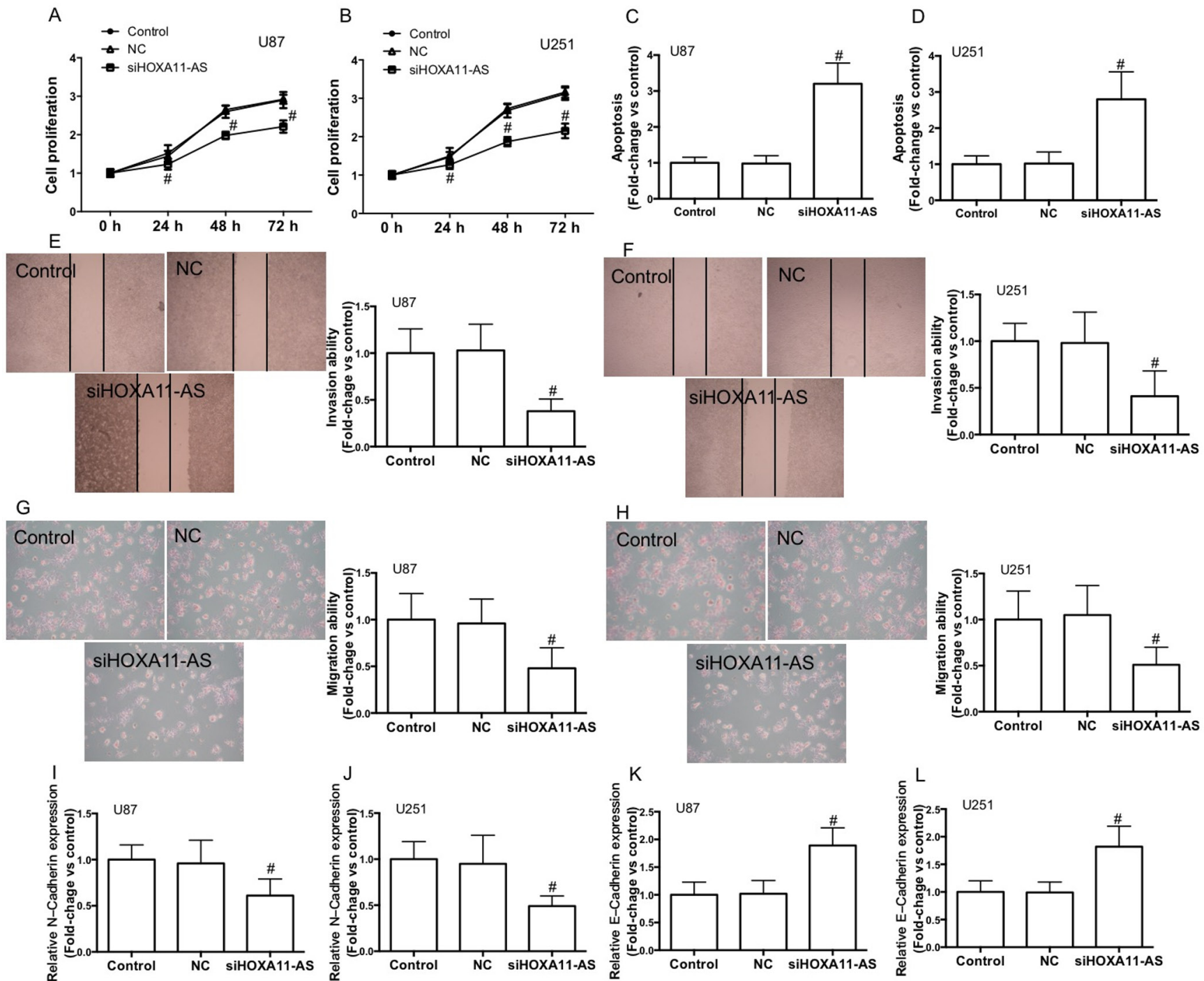

Figure 3. Effects of HOXA11-AS silencing on glioma cell proliferation, apoptosis, invasion, and migration in vitro. U87 and U251 cells were transfected with siHOXA11-AS or control siRNAs. (A and B) Cell proliferation was evaluated by CCK8 kit. (C and D) Apoptosis was measured by Tunel staining and analyzed by flow cytometry. Results are shown as fold-change vs control. (E and F) Invasion ability was evaluated by Transwell assay and results are shown as fold-change vs control. ( $\mathrm{G}$ and $\mathrm{H}$ ) Migration ability was evaluated by Wound healing assay and results are shown as fold-change vs control. (I and J) mRNA expression of N-Cadherin was determined by qRT-PCR. (K and L) mRNA expression of E-Cadherin was determined by qRT-PCR. $\# \mathrm{p}<0.05$, compared with control.

LncRNA HOXA11-AS expression, the malignancy of glioma exhibits an increasing tendency. To further examine the correlation of LncRNA HOXA11-AS expression with glioma patients prognosis, Kaplan-Meier analysis and log-rank test were performed. As shown in Figure 1C, patients with high LncRNA HOXA11-AS expression had shorter OS time than those with low LncRNA HOXA11-AS expression $(\mathrm{HR}=1.849$, $95 \%$ confidence interval $(C I)=1.219-3.872, p=0.0096)$. These results suggest that upregulation of LncRNA HOXA11-AS might serve as a prognostic marker for glioma patients.

Silencing LncRNA HOXA11-AS expression in glioma cells by siRNA. To investigate the functional role of LncRNA HOXA11-AS in glioma cells, qRT-PCR was conducted to measure the expression of LncRNA HOXA11-AS in normal brain tissues and three glioma cell lines, including T98G, U87, and U251 cell lines. As shown in Figure 2A, the expression of LncRNA HOXA11-AS was significantly higher in three high-degree glioblastoma cell lines (T98G, U87, and U251) compared to normal brain tissues. U87 and U251 cells were transfected with siHOXA11-AS and the qRT-PCR results confirmed the efficiency of the HOXA11-AS interference (Figure 2B and C).

Silencing LncRNA HOXA11-AS inhibits cell proliferation, increases apoptosis, and inhibits invasion and migration of glioma cells. To further examine whether LncRNA HOXA11-AS was involved in glioma progression, glioma 
A

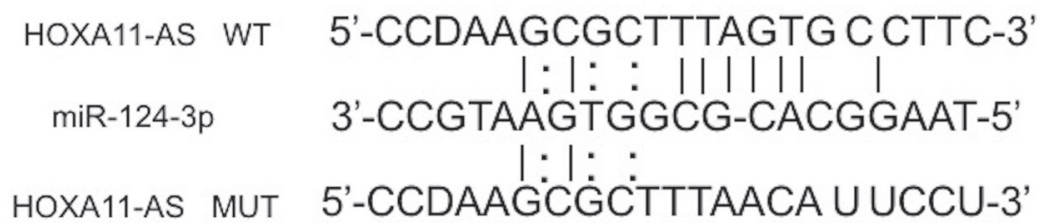

B

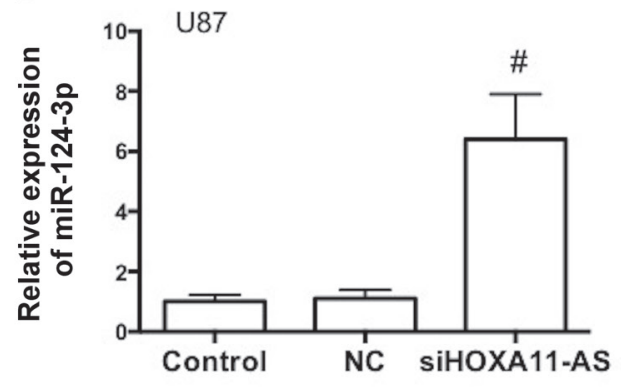

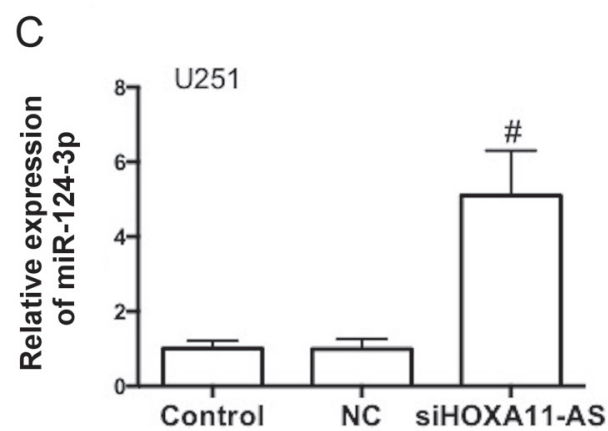

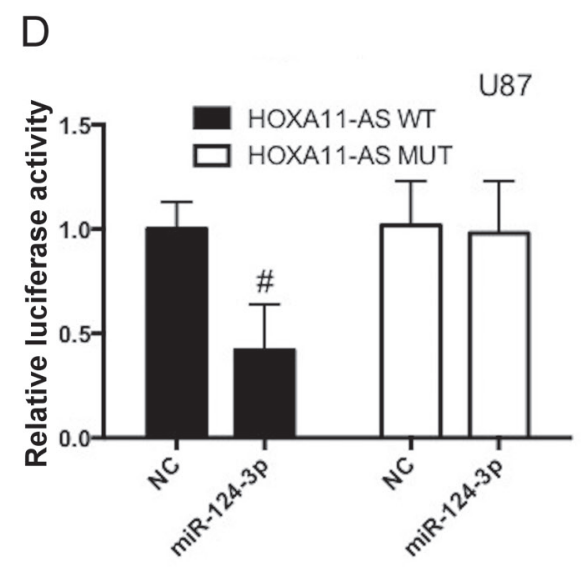

$\mathrm{E}$

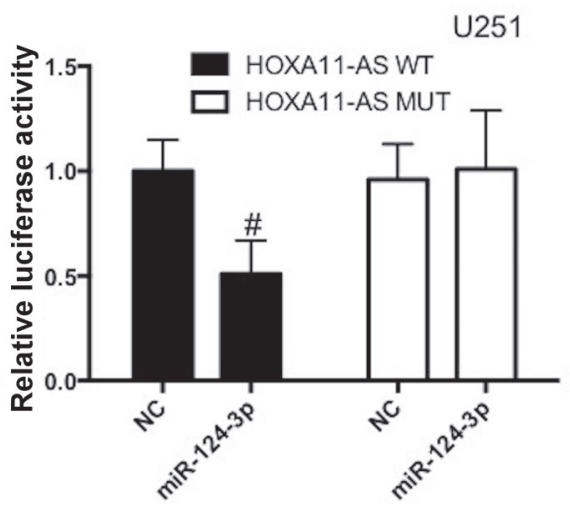

$\mathrm{F}$

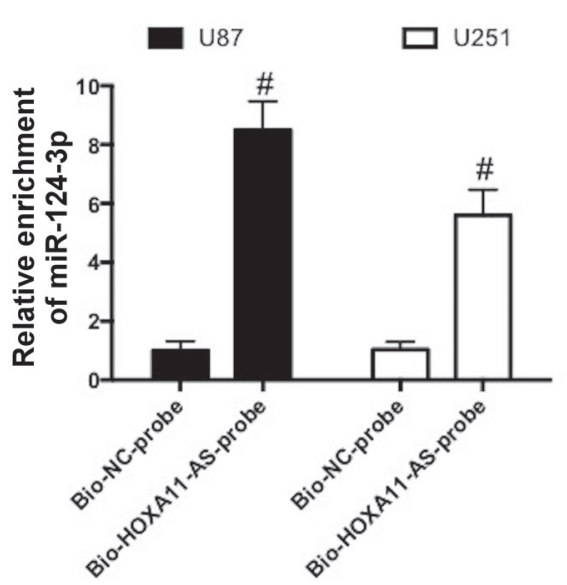

Figure 4. LncRNA HOXA11-AS acts as a molecular sponge for miR-124-3p in glioma cells. (A) The predicted miR-124-3p binding sites on HOXA11AS. (B and C) U87 and U251 cells were transfected with siHOXA11-AS or control siRNAs. miR-124-3p was determined by qRT-PCR. (D and E) U87 and U251 cells were co-transfected with miR-124-3p mimics and luciferase reporters containing HOXA11-AS-WT or HOXA11-AS-MUT vector and luciferase activities were determined by assay kits. (F) miR-124-3p detection using qRT-PCR in the sample pulled down by biotinylated HOXA11-AS probe. $\# \mathrm{p}<0.05$, compared with control.

malignancy was evaluated. In both U87 and U251 cell lines, cell proliferation was reduced by siHOXA11-AS (Figure 3A and $\mathrm{B})$ which was detected by CCK8 assay kit. In addition, silencing HOXA11-AS significantly increased the apoptotic cell folds (Figure 3C and D). Moreover, cell invasion ability was investigated by Transwell invasion assay. We showed that the invasion capacities of U87 and U251 cells transfected with siHOXA11-AS were significantly reduced compared to the siNC group (Figure 3E and F). Furthermore, migration ability was investigated by Wounding healing assay. The results show that migration capacities of U87 and U251 cells transfected with siHOXA11-AS were significantly decreased compared to the siNC group (Figure $3 \mathrm{G}$ and $\mathrm{H}$ ). N-Cadherin expression was significantly reduced by siHOXA11-AS in U87 and U251 cells (Figure 3I and J). siHOXA11-AS notably increased E-Cadherin expression in U87 and U251 cells (Figure $3 \mathrm{~K}$ and $\mathrm{L}$ ). These results demonstrate that LncRNA HOXA11-AS significantly promotes cell growth, invasion and migration of glioma cells.

HOXA1 1-AS acts as a molecular sponge for miR-124-3p. LncRNAs could function as ceRNAs to specific miRNAs. In our study, we observed that miR-124-3p had relevant binding sites in HOXA11-AS using the online software starBase v2.0 (Figure 4A). Silencing HOXA11-AS significantly increased miR-124-3p expression in U87 and U251 cells (Figure 4B and C). The results of dual-luciferase reporter assay revealed that 

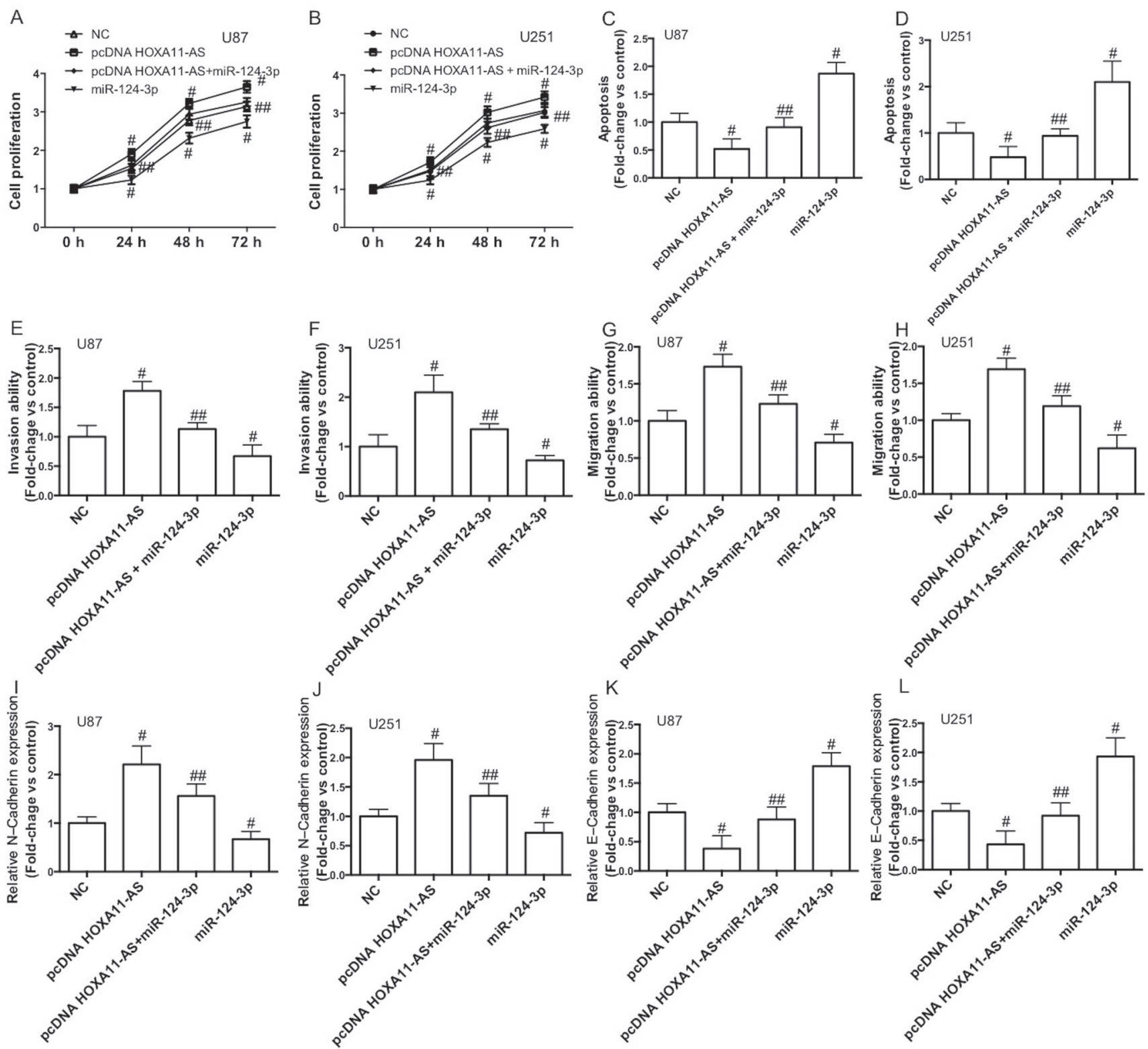

Figure 5. LncRNA HOXA11-AS promotes glioma cell malignancy by competitively binding miR-124-3p. U87 and U251 cells were co-transfected with pcDNA HOXA11-AS with or without miR-124-3p mimics. (A and B) Cell proliferation was evaluated by CCK8 kit. (C and D) Apoptosis was measured by Tunel staining and analyzed by flow cytometry. Results are shown as fold-change vs control. (E and F) Invasion ability was evaluated by Transwell assay and results are shown as fold-change vs control. ( $\mathrm{G}$ and $\mathrm{H})$ Migration ability was evaluated by Wound healing assay and results are shown as foldchange vs control. (I and J) mRNA expression of N-Cadherin was determined by qRT-PCR. (K and L) mRNA expression of E-Cadherin was determined by $\mathrm{qRT}$-PCR. \#p $<0.05$, compared with control. \#\#p<0.05, compared with pcDNA HOXA11-AS treatment.

miR-124-3p overexpression decreased the luciferase activity of the pMIR luciferase reporter containing HOXA11-AS-WT but not HOXA11-AS-MUT (Figure 4D and E). Moreover, we performed a pull down assay using biotin-labeled specific HOXA11-AS probe. We found that miR-124-3p was precipitated as revealed by qRT-PCR (Figure 4F). The results indicate that HOXA11-AS acts as a molecular sponge for miR-124-3p.
MiR-124-3p reverses the LncRNA HOXA11-AS effects on the malignancy of glioma cells. To further elucidate the binding role of the miR-124-3p with LncRNA HOXA11AS in the progression of glioma, we increased miR-124-3p in pcDNA-HOXA11-AS transfected glioma cells. We show that overexpression of LncRNA HOXA11-AS increased cell proliferation in U87 and U251 cell lines, while miR-124-3p mimics decreased cell proliferation (Figure $5 \mathrm{~A}$ and $\mathrm{B}$ ). 
A

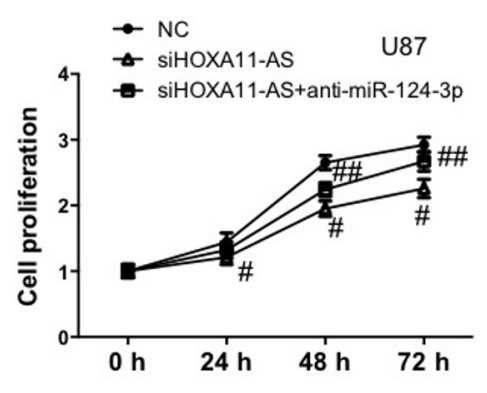

B

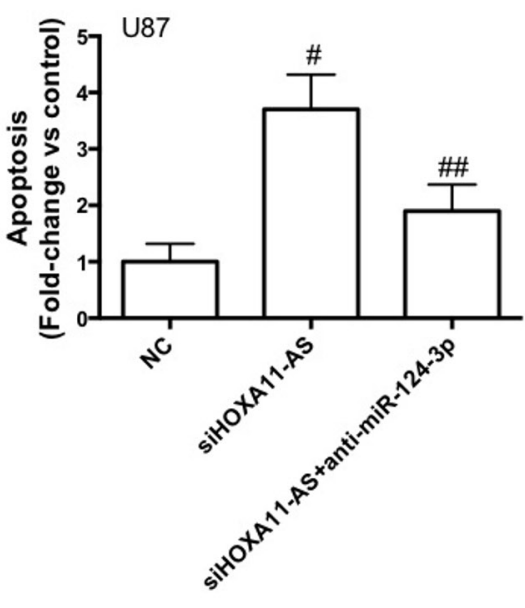

E

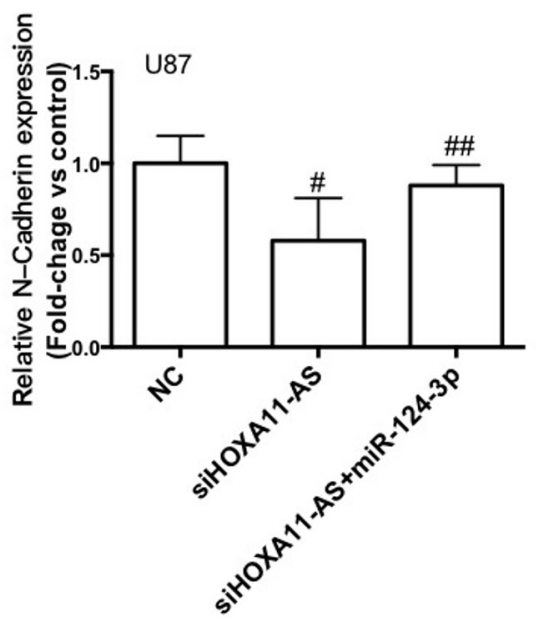

C

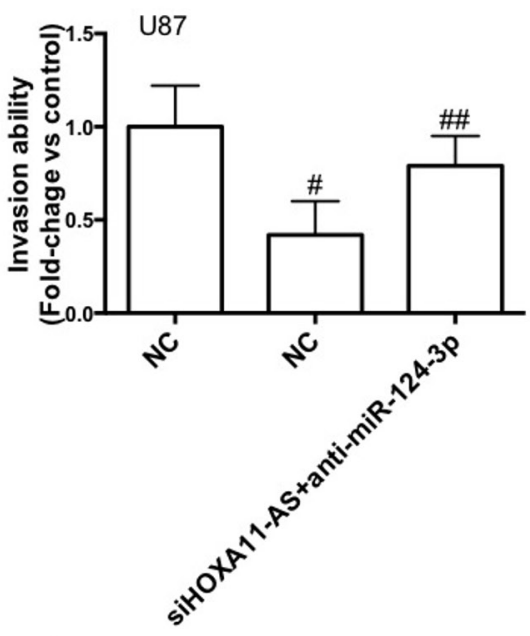

$\mathrm{F}$

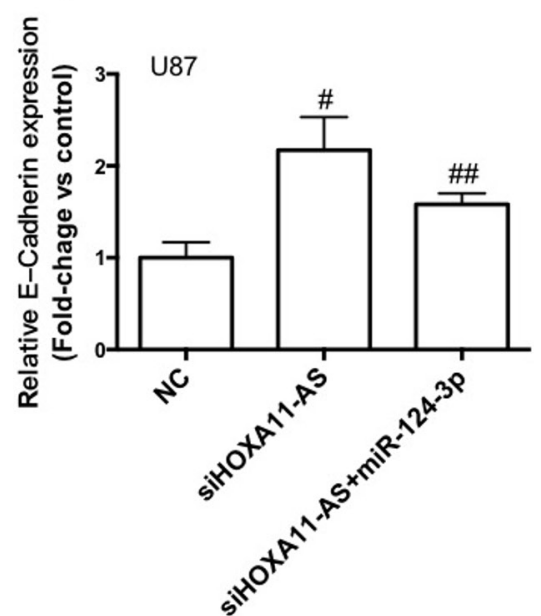

Figure 6. Inhibition of miR-124-3p suppressed HOXA11-AS silence-induced inhibition of glioma cell malignancy. U87 cells were transfected with siHOXA11-AS with or without anti-miR-124-3p. (A) Cell proliferation was evaluated by CCK8 kit. (B) Apoptosis was measured by Tunel staining and analyzed by flow cytometry. Results are shown as fold-change vs control. (C) Invasion ability was evaluated by Transwell assay and results are shown as fold-change vs control. (D) Migration ability was evaluated by Wound healing assay and results are shown as fold-change vs control. (E) $\mathrm{mRNA}$ expression of N-Cadherin was determined by qRT-PCR. (F) mRNA expression of E-Cadherin was determined by qRT-PCR. \#p $<0.05$, compared with control. $\#$ \# $<0.05$, compared with siDNA HOXA11-AS treatment.

miR-124-3p mimics significantly reversed the promoting effect of LncRNA HOXA11-AS on cell proliferation (Figure 5A and B). Overexpression of LncRNA HOXA11AS decreased apoptosis in U87 and U251 cell lines, while miR-124-3p mimics increased apoptosis (Figure 5C and D). The miR-124-3p mimics significantly reversed the inhibitory effect of LncRNA HOXA11-AS on apoptosis (Figure 5C and D). the LncRNA HOXA11-AS overexpression increased the invasion ability in U87 and U251 cell lines, while miR-124-3p mimics decreased it (Figure 5E and F). miR-124-3p mimics significantly reversed the promoting effect of LncRNA HOXA11-AS on invasion ability (Figure 5E and F). Moreover, overexpression of LncRNA HOXA11-AS increased migration ability in U87 and U251 cell lines, while miR-124-3p mimics decreased migration ability (Figure $5 \mathrm{G}$ and $\mathrm{H}$ ). miR-124-3p mimics significantly reversed the promoting effect of LncRNA HOXA11-AS on migration ability (Figure $5 \mathrm{G}$ and $\mathrm{H})$. Overexpression of LncRNA HOXA11-AS increased N-Cadherin expression in U87 and U251 cell lines, while miR-124-3p mimics decreased N-Cadherin expression (Figure 5I and J). miR-124-3p mimics significantly reversed the increasing effect of LncRNA HOXA11AS on N-Cadherin expression (Figure 5I and J). Overexpression of LncRNA HOXA11-AS decreased E-Cadherin expression in U87 and U251 cell lines, while miR-124-3p mimics increased E-Cadherin expression (Figure $5 \mathrm{~K}$ and L). miR-124-3p mimics significantly reversed the decreasing effect of LncRNA HOXA11-AS on E-Cadherin expression 
(Figure 5K and L). Furthermore, we transfected glioma cells with siDNA-HOXA11-AS and anti-miR-124-3p and revealed that miR-124-3p inhibitor significantly reversed the siHOXA11-AS inhibitory effect on cell proliferation (Figure 6A), apoptosis (Figure 6B), invasion ability (Figure 6C), migration ability (Figure 6D), N-Cadherin expression (Figure 6E) and E-Cadherin expression (Figure 6F). Inhibition of miR-124-3p suppressed HOXA11-AS silence-induced inhibition of glioma cell malignancy. These results highlight the role of the LncRNA HOXA11-AS binding to miR-124-3p in glioma growth, invasion and migration.

\section{Discussion}

HOXA11-AS, with a length of 3885 nucleotides, a member of the HOX family gene, is located on 7p15.2 [23]. Recent evidence has proved that HOXA11-AS is critical for several types of tumor carcinogenesis and progression. Overexpression of LncRNA HOXA11-AS promotes cell epithelial-mesenchymal transition by repressing miR-200b in non-small cell lung cancer [24]. LncRNA HOXA11-AS promotes proliferation and invasion of gastric cancer by scaffolding the chromatin modification factors PRC2, LSD1, and DNMT1 [25]. LncRNA HOXA11-AS induces tumor progression and stemness maintenance in cervical cancer [26]. Moreover, Wang et al. found that LncRNA HOXA11AS was increased in glioma tissues and could promote gliomagenesis by regulating cell cycle progression [27]. Nevertheless, the expression and underlying mechanism of HOXA11-AS in glioma progression is still unclear and not well understood.

In the present study, we found that LncRNA HOXA11-AS expression was markedly elevated in glioma tissues compared to normal brain tissues. The LncRNA HOXA11-AS expression in cases of high-grade glioma was significantly higher than that in cases of low-grade. Patients with high LncRNA HOXA11-AS expression had shorter OS time than those with low LncRNA HOXA11-AS expression. Moreover, silencing LncRNA HOXA11-AS inhibited cell proliferation, increased apoptosis, and inhibited invasion and migration of glioma cells. LncRNA HOXA11-AS overexpression increased cell proliferation, decreased apoptosis, and increased invasion and migration of glioma cells. These results suggest that upregulation of LncRNA HOXA11-AS is associated with the glioma malignancy and might serve as a prognostic marker for glioma patients.

Recently, it has been shown that LncRNAs could regulate tumor progression by functioning as ceRNAs to specific miRNAs $[28,29]$. MicroRNAs (miRNAs, $22 \mathrm{nt}$ ) are a group of small non-coding RNAs that are involved in diverse biological processes, such as cell growth, migration, apoptosis and differentiation, by binding to the 3'-UTRs of target genes $[30,31]$. We observed that $\mathrm{miR}-124-3 \mathrm{p}$ had relevant binding sites in HOXA11-AS. Silencing HOXA11-AS significantly increased miR-124-3p expression. miR-124-3p overexpression decreased the luciferase activity of the pMIR luciferase reporter containing HOXA11-AS-WT but not HOXA11-ASMUT. Moreover, we found that miR-124-3p was pulled down by HOXA11-AS probe. The results indicate that HOXA11-AS acts as a molecular sponge for miR-124-3p. In osteosarcoma, it has been found that LncRNA HOXA11-AS functions as a competing endogenous RNA to regulate ROCK1 expression by sponging miR-124-3p [32]. Eguia-Aguilar et al. reported that reduction of the miR-124-3p expression in pediatric astrocytomas was related to high-grade supratentorial and recurrent tumors in Mexican children [33]. Suzhi reported that miR-124-3 exhibited anti-proliferative effect in glioblastoma cells [34]. miR-124-3p regulates cell proliferation, invasion, apoptosis, and bioenergetics by targeting PIM1 in astrocytoma [35]. In the current study, we found that miR-124-3p mimics inhibited cell proliferation, increased apoptosis, and inhibited invasion and migration of glioma cells. miR-124-3p mimics significantly suppressed overexpression of HOXA11-AS-induced increase of proliferation, decrease of apoptosis and increase of invasion and migration. miR-124-3p inhibitors significantly suppressed silencing of HOXA11-AS-induced decrease of proliferation, increase of apoptosis and decrease of invasion and migration. Further studies are needed to identify the downstream regulators of LncRNA HOXA11-AS/miR-124-3p axis in the regulation of glioma progression.

In summary, these findings highlight the importance of LncRNA HOXA11-AS/miR-124-3p axis in the regulation of glioma progression. LncRNA HOXA11-AS/miR-124-3p might serve as a potential therapeutic target in the glioma treatment in the future.

\section{References}

[1] SIEGEL RL, MILLER KD, JEMAL A. Cancer statistics, 2016. CA Cancer J Clin 2016; 66: 7-30. https://doi.org/10.3322/ caac. 21332

[2] SHERMANJH, HOES K, MARCUSJ, KOMOTAR RJ, BRENNAN CW et al., Neurosurgery for brain tumors: update on recent technical advances. Curr Neurol Neurosci Rep 2011; 11: 313-319. https://doi.org/10.1007/s11910-011-0188-9

[3] WEN PY, KESARI S. Malignant gliomas in adults. N Engl J Med 2008; 359: 492-507. https://doi.org/10.1056/NEJMra0708126

[4] SATHORNSUMETEE S, RICH JN. New treatment strategies for malignant gliomas. Expert Rev Anticancer Ther 2006; 6: 1087-1104. https://doi.org/10.1586/14737140.6.7.1087

[5] TSAI MC, SPITALE RC, CHANG HY. Long intergenic noncoding RNAs: new links in cancer progression. Cancer Res 2011; 71: 3-7. https://doi.org/10.1158/0008-5472.CAN-102483

[6] SHI XM, TENG F. Up-regulation of long non-coding RNA Sox2ot promotes hepatocellular carcinoma cell metastasis and correlates with poor prognosis. Int J Clin Exp Pathol 2015; 8: 4008-4014. 
[7] VILLAMIZAR O, CHAMBERS CB, MO YY, TORRY DS, HOFSTRAND R et al. Data in support of transcriptional regulation and function of Fas-antisense long noncoding RNA during human erythropoiesis. Data Brief 2016; 7: 1288-1295. https://doi.org/10.1016/j.dib.2016.03.106

[8] LI JK, CHEN C, LIU JY, SHI JZ, LIU SP et al. Long noncoding RNA MRCCAT1 promotes metastasis of clear cell renal cell carcinoma via inhibiting NPR3 and activating p38-MAPK signaling. Mol Cancer 2017; 16: 111. https://doi. org/10.1186/s12943-017-0681-0

[9] ZOU G1, LIU T2, GUO L3, HUANG Y4, FENG Y et al. miR-145 modulates lncRNA-ROR and Sox2 expression to maintain human amniotic epithelial stem cell pluripotency and beta islet-like cell differentiation efficiency. Gene 2016; 591: 48-57. https://doi.org/10.1016/j.gene.2016.06.047

[10] JIANG L, LIU W, ZHU A, ZHANG J, ZHOU J et al. Transcriptome analysis demonstrate widespread differential expression of long noncoding RNAs involve in Larimichthys crocea immune response. Fish Shellfish Immunol 2016; 51: 1-8. https://doi.org/10.1016/j.fsi.2016.02.001

[11] YANG D, SUN L, LI Z, GAO P. Noncoding RNAs in Regulation of Cancer Metabolic Reprogramming. Adv Exp Med Biol 2016; 927: 191-215. https://doi.org/10.1007/978-98110-1498-7_7

[12] DONG R, LIU GB, LIU BH, CHEN G, LI K et al. Targeting long non-coding RNA-TUG1 inhibits tumor growth and angiogenesis in hepatoblastoma. Cell Death Dis 2016; 7: e2278. https://doi.org/10.1038/cddis.2016.143

[13] BARTONICEK N, MAAG JL, DINGER ME. Long noncoding RNAs in cancer: mechanisms of action and technological advancements. Mol Cancer 2016; 15: 43. https://doi. org/10.1186/s12943-016-0530-6

[14] CLOUTIER SC, WANG S, MA WK, AL HUSINI N, DHOONDIA Z et al. Regulated Formation of lncRNA-DNA Hybrids Enables Faster Transcriptional Induction and Environmental Adaptation. Mol Cell 2016; 62: 148. https://doi. org/10.1016/j.molcel.2016.03.012

[15] LIU Q, GUO X, QUE S, YANG X, FAN H et al. LncRNA RSU1P2 contributes to tumorigenesis by acting as a ceRNA against let-7a in cervical cancer cells. Oncotarget 2016; 8: 43768-43781. https://doi.org/10.18632/oncotarget.10844

[16] ELLINGER J, ALAM J, ROTHENBURG J, DENG M, SCHMIDT D et al. The long non-coding RNA lnc-ZNF180-2 is a prognostic biomarker in patients with clear cell renal cell carcinoma. Am J Cancer Res 2015; 5: 2799-2807.

[17] JIANG CY, GAO Y, WANG XJ, RUAN Y, BEI XY et al. Long non-coding RNA lnc-MX1-1 is associated with poor clinical features and promotes cellular proliferation and invasiveness in prostate cancer. Biochem Biophys Res Commun 2016; 470: 721-727. https://doi.org/10.1016/j.bbrc.2016.01.056

[18] NUNES FD, DE ALMEIDA FC, TUCCI R, DE SOUSA SC. Homeobox genes: a molecular link between development and cancer. Pesqui Odontol Bras 2003; 17: 94-98.

[19] DEAR TN, SANCHEZ-GARCIA I, RABBITTS TH. The HOX11 gene encodes a DNA-binding nuclear transcription factor belonging to a distinct family of homeobox genes. Proc Natl Acad Sci U S A 1993; 90: 4431-4435.
[20] BHATLEKAR S, FIELDS JZ, BOMAN BM. HOX genes and their role in the development of human cancers. J Mol Med (Berl) 2014; 92: 811-823. https://doi.org/10.1007/s00109014-1181-y

[21] Li T, et al. Expression and clinicopathological significance of the lncRNA HOXA11-AS in colorectal cancer. Oncol Lett 2016; 12: 4155-4160. https://doi.org/10.3892/ol.2016.5129

[22] LI T, XU C, CAI B, ZHANG M, GAO F et al. Over-expressed long noncoding RNA HOXA11-AS promotes cell cycle progression and metastasis in gastric cancer. Mol Cancer, 2017. 16(1): p. 82. https://doi.org/10.3892/ol.2016.5129

[23] RICHARDS EJ, PERMUTH-WEY J, LI Y, CHEN YA, COPPOLA D et al. A functional variant in HOXA11-AS, a novel long non-coding RNA, inhibits the oncogenic phenotype of epithelial ovarian cancer. Oncotarget 2015; 6: 34745-34757. https://doi.org/10.18632/oncotarget.5784

[24] CHEN JH, ZHOU LY, XU S, ZHENG YL, WAN YF et al. Overexpression of IncRNA HOXA11-AS promotes cell epithelial-mesenchymal transition by repressing miR-200b in non-small cell lung cancer. Cancer Cell Int 2017; 17: 64. https://doi.org/10.1186/s12935-017-0433-7

[25] SUN M, NIE F, WANG Y, ZHANG Z, HOU J et al. LncRNA HOXA11-AS Promotes Proliferation and Invasion of Gastric Cancer by Scaffolding the Chromatin Modification Factors PRC2, LSD1, and DNMT1. Cancer Res 2016; 76: 6299-6310. https://doi.org/10.1158/0008-5472.CAN-16-0356

[26] KIM HJ, EOH KJ, KIM LK, NAM EJ, YOON SO et al. The long noncoding RNA HOXA11 antisense induces tumor progression and stemness maintenance in cervical cancer. Oncotarget 2016; 7: 83001-83016. https://doi.org/10.18632/ oncotarget.12863

[27] WANG Q, ZHANG J, LIU Y, ZHANG W, ZHOU J et al. A novel cell cycle-associated lncRNA, HOXA11-AS, is transcribed from the 5-prime end of the HOXA transcript and is a biomarker of progression in glioma. Cancer Lett 2016; 373: 251-259. https://doi.org/10.1016/j.canlet.2016.01.039

[28] GONG W, ZHENG J, LIU X, MA J, LIU Y et al. Knockdown of NEAT1 restrained the malignant progression of glioma stem cells by activating microRNA let-7e. Oncotarget 2016; 7: 62208-62223. https://doi.org/10.18632/oncotarget.11403

[29] LV QL, CHEN SH, ZHANG X, SUN B, HU L et al. Upregulation of long noncoding RNA zinc finger antisense 1 enhances epithelial-mesenchymal transition in vitro and predicts poor prognosis in glioma. Tumour Biol 2017; 39: 1010428317695022. https://doi. org/10.1177/1010428317695022

[30] FRIXA T, DONZELLI S, BLANDINO G. Oncogenic MicroRNAs: Key Players in Malignant Transformation. Cancers (Basel) 2015; 7: 2466-2485. https://doi.org/10.3390/ cancers7040904

[31] WANG P, LIU YH, YAO YL, LI Z, LI ZQ et al. Long noncoding RNA CASC2 suppresses malignancy in human gliomas by miR-21. Cell Signal 2015; 27: 275-282. https://doi. org/10.1016/j.cellsig.2014.11.011

[32] CUI M, WANG J, LI Q, ZHANG J, JIA J et al. Long noncoding RNA HOXA11-AS functions as a competing endogenous RNA to regulate ROCK1 expression by sponging miR-124-3p in osteosarcoma. Biomed Pharmacother 2017; 92: 437-444. https://doi.org/10.1016/j.biopha.2017.05.081 
[33] EGUIA-AGUILAR P, PEREZPENA-DIAZCONTI M, BENADON-DARSZON E, CHICO-PONCE DE LEON F, GORDILLO-DOMINGUEZ L et al. Reductions in the expression of miR-124-3p, miR-128-1, and miR-221-3p in pediatric astrocytomas are related to high-grade supratentorial, and recurrent tumors in Mexican children. Childs Nerv Syst 2014; 30: 1173-1181. https://doi.org/10.1007/s00381-0142416-5
[34] SUZHI Z, LIANG T, YUEXIA P, LUCY L, XIAOTING H et al. Gap Junctions Enhance the Antiproliferative Effect of MicroRNA-124-3p in Glioblastoma Cells. J Cell Physiol 2015; 230: 2476-2488. https://doi.org/10.1002/jcp.24982

[35] DENG D, WANG L, CHEN Y, LI B, XUE L et al. MicroRNA124-3p regulates cell proliferation, invasion, apoptosis, and bioenergetics by targeting PIM1 in astrocytoma. Cancer Sci 2016; 107: 899-907. https://doi.org/10.1111/cas.12946 